\title{
Route analysis in complex buildings
}

\author{
Alexander Koutamanis ${ }^{1}{ }^{2}$, Marc van Leusen ${ }^{1}$ and Vicky Mitossi ${ }^{1}$ \\ Government Buildings Agency, Ministry of Housing, Spatial Planning and the Environment, The \\ Hague $^{1}$, Faculty of Architecture, Delft University of Technology ${ }^{2}$
}

Key words: pedestrian circulation, analysis, representation, interaction

\begin{abstract}
Analysis of pedestrian circulation in buildings is usually performed in the early stages of the design process or later on with respect to a specific design problem such as fire safety. In both cases, the analysis relates more to wayfinding, i.e. search for a route on the basis of fundamental normative criteria. Wayfinding analysis in existing buildings is useful for the comparison between "rational" behaviour and actual usage but this comparison does little to explicate the observed structure of pedestrian circulation. In contrast to wayfinding, route analysis deals with the registration and assessment of actual patterns of pedestrian circulation in existing buildings. These patterns are represented topologically and geometrically. The geometric representation makes use of norms underlying building codes in order to reach an appropriate level of abstraction. Route representations are implemented on top of a building representation of relevant spatial and building elements. The building representation serves both as input and output for the route analysis. Input and output are largely automated, including production of the geometric route locally (i.e. within each space) and measurement of route distance and complexity. Use data are collected in an alphanumeric database and linked dynamically to the geometric and topological representation. Route analysis supports and refines other forms of post-occupancy evaluation by adding important dynamic aspects to activity allocation and compartmentalization.
\end{abstract}

\section{PEDESTRIAN CIRCULATION IN BUILDINGS}

It has been suggested that as a technological field matures its development gradually shifts from illustrations of fundamental ideas to demonstrations of how problems of practical significance can be solved and then to experiments that attempt to solve problems of practical interest 
(Winston, 1992). Despite the extent of the ongoing digitization of social processes and professional services, the computerization of architecture especially in practice- still deals primarily with illustrations. One of the main problems in current developments is the limited integration, utilization and enrichment of domain knowledge. Human interaction with the built environment has been one of the knowledge components that are frequently underplayed in research, development and practice. The reasons for doing so include the domination of normative approaches to design and analysis (Koutamanis and Mitossi, 1996; Koutamanis, 2000), the complexity of human interaction, the aesthetization of architectural design and the lack of knowledge transfer in particular from cognitive science. In particular, dynamic aspects of the use of the built environment remain largely uncharted despite the ready availability of basic information on the cognitive, ergonomic and other dimensions of such aspects. One of these aspects is pedestrian circulation in buildings, which ironically is a primary even though frequently implicit factor in a variety of applications, such as the clustering and allocation of activities in a building (as in space allocation and programmatic analysis), code compliance (e.g. with respect to fire safety) and cost estimation.

Reasons for the relative neglect of pedestrian circulation in architectural computerization include:

- The complexity of dynamic human interaction with buildings: in moving from one space to another we process a wide spectrum of information, taking a large number of decisions and performing conscious and unconscious actions relating not only to the purpose of the movement but also to different contexts at the same time.

- The complexity of computer simulations: simulations for reproducing dynamic human interaction with buildings require high precision and accuracy, as well as computational power for implementation, testing and use.

- Lack of comprehensive, integrated data on dynamic human interaction with buildings: despite the availability of technologies for capturing human movement and the extent of relevant cognitive and ergonomic research, we possess no unified, verifiable body of data that could drive computer simulations of human movement in the built environment.

- The relative weakness of programmatic and functional analysis: the building brief is normally insufficient for design guidance with respect to pedestrian circulation or for the analysis and evaluation of designs produced on the basis of the brief. This is accentuated further by the normative abstraction of building codes and general professional guidelines. Most analyses of designs and programmes appear to accept the reductive logic of such codes and guidelines. 
- Lack of integration with design synthesis: the representations and other data used in analysis are frequently kept separate from the instruments and environments used in synthesis. As a result, providing input to the analysis becomes a tedious, redundant task. Even more important is the lack of direct feedback that constrains the further development of a design. Therefore, the designer currently views computational design analysis as a cumbersome, time-consuming alternative to intuitive analysis.

Our work focuses on the last two reasons. In the framework of the large, complex institutional buildings designed and managed by the Government Buildings Agency, conventional practices are frequently dismissed by all involved parties on the grounds that they are grossly insufficient for an accurate, precise and reliable projection of building behaviour and performance, without which it is impossible to take reasonable, defendable decisions. The extensive experience of clients, users and designers with the specific problems of governmental and institutional buildings makes the oversimplifications of intuitive and normative reasoning too weak to accept as fundamental common points of departure. At the same time, this experience provides a comprehensive (even though rather incoherent) empirical basis for the development of practical methods and instruments that make designing and communication efficient and transparent.

The simulation and analysis of a dynamic aspect such as pedestrian circulation relies on a representation consisting of a number of interrelated components:

1. Actor(s): $\operatorname{person}(\mathrm{s})$ who travel(s)

2. Starting point: the location from where the actor(s) depart. In buildings this location can be a space, a typical point in the departure space (such as its centroid) or a doorway. Multiple starting points indicate aggregation of routes / travels.

3. Destination: the location where the actor(s) go. Multiple destinations are not necessarily product of aggregation: a route / travel may also contain intermediate destinations. Also attractors such as stairs, lifts and control points can be treated as intermediate destinations.

4. Path: starting point, destination and locations traversed between the two. The path can be either the actual path of the route or an approximation of it (straight line or city block).

5. Means of transportation: how locomotion is performed along the path, including speed and capacity achieved by these means.

6. Activities that take place along the path:

a. Activities related to transportation

b. Intervening opportunities, such as relations to other routes or other activities and actors in the building 
The complexity and extent of the information in such a representation, as well as the corresponding tenacity of the analysis or simulation, mean that analysis of pedestrian circulation in buildings is usually performed either in the early stages of the design process when information and demands are quite abstract or later on with respect to some concrete design problem such as fire safety (Ozel, 1992). In both cases, the analysis relates more to wayfinding, i.e. search for a route on the basis of fundamental criteria such as the norms of building rules and regulations and basic contextual information. The ways the building is experienced, explored, understood and remembered by its users and occupants remains largely unexplored, even though it is an important factor in e.g. egress behaviour (Marchant, 1980; Shields and Silcock, 1987; Stollard and Abrahams, 1995). The reasons for the relative neglect of post-occupancy pedestrian circulation in research and practice are quite pragmatic. In addition to the already mentioned problems of complexity and lack of basic data, architecture deals with constraints that tend to be too abstract, as in code compliance. Detailed registration and analysis is therefore deemed useful only for the derivation of general principles and guidelines. This is accentuated by the lack of specificity in the form of a design and in the activities of its users in most design stages.

Wayfinding analysis in existing buildings is useful for the comparison between "rational" (normative) behaviour and actual usage, as well as for the refinement of predictive models of wayfinding for architectural design (Gross and Zimring, 1992; Shih and Yan, 1997). However, this comparison is generally transparent at the level of general principles or in very specific situations but as yet can do little to explicate the observed overall structure of pedestrian circulation, especially in large buildings. Recent extensions of basic wayfinding with techniques characterized by autonomy promise a correlation between local conditions and global structure. At low dimensionality (i.e. mostly concerned with internal interactions between pedestrians and flows) and at relatively high abstraction (urban design and higher) they provide useful insights into the potential of autonomous mechanisms in circulation simulation (Blue and Adler, 1998; Dijkstra, Timmermans et al., 2000). However, the complexity of interaction in real buildings appears to be too high for effective and reliable solutions yet.

Arguably the strongest point and at the same time the most important limitation of recent analyses and simulations of wayfinding behaviour lies in their attention for local decisions and actions. This is apparently a reaction to the determinism and reductionism of normative approaches that promises to enrich our understanding of cognitive factors in functional aspects and can lead to technical improvement in simulation and analysis. At the same time, attention for local decisions and conditions may obscure global characteristics of a route, relations between routes and the conscious 
adaptation of routes for extrinsic reasons, such as in the arrangement of a travelling exposition in different exposition spaces or arbitrary compartmentalization of a building for security reasons. The current tendency of considering global dynamic patterns as mere products of local ones does little credit to both human adaptability and design capacity.

\section{AN APPROACH TO ROUTE ANALYSIS}

Our work follows a pragmatic bottom-up approach, intended to build on and enrich gradually existing professional practices. We employ a standard commercial CAD program (AutoCAD) for inputting and manipulating design representations that come close to conventional CAD drawings (Mitossi and Koutamanis, 1998). The use of these representations in design and analysis is structured and facilitated by a number of add-on modules (in Lisp and Visual Basic) for extracting information from the drawing representation, for performing analyses automatically and for checking grammatical or syntactic consistency and completeness (Koutamanis and Mitossi, 2000). The basic building representation consists of:

- Spaces: implemented in $2 \frac{1}{2} \mathrm{D}$ as polygonal outlines with a height indication (lightweight polylines with thickness)

- Building elements: also implemented in $2 \frac{1}{2} \mathrm{D}$ similarly to the spaces

- A layer system for the modularization of design information: clustering of spaces and building element by floor level and type

- Alphanumeric annotations on the spaces and building elements: these indicate properties that cannot be indicated graphically and are implemented as dynamic links to databases (in MS Access)

Relations between elements of the representation are recognized on an ifneeded basis: when a control action or an analysis require information on e.g. relationships of inclusion or adjacency, the representation activates local detection routines that test specific relevant conditions. Most detection results are registered only temporarily in the framework of feedback mechanisms but often trigger other actions, such as a correction of the geometry of a space or building element. Automatic detection of relations and properties of spaces and building elements form the basis of most analyses. These are generally exported to prepared databases and spreadsheets (in MS Excel) where the actual analysis takes place (Leusen and Mitossi, 1998). The dynamic information system that comprises the representations, analyses and related background information, such as the building programme and cost data, is implemented as a Web-based compound structure complete with communication facilities for use in the Intranet of the Government Buildings Agency or project-based Extranets. 
In the framework of earlier research projects the analysis of pedestrian circulation has been considered with respect to the general structure of the design representation, as well as for specific functional issues (Koutamanis and Mitossi, 1993; Koutamanis, 1995). In accordance with our descriptive approach to design and analysis, these projects attempted to complement and question normative levels with a more detailed and accurate registration or projection of human interaction with the built environment.

In contrast to the predictions made in wayfinding analysis, route analysis deals with the registration and assessment of actual patterns of pedestrian circulation in existing buildings. The complex situations we experience in large, sensitive buildings such as prisons and law courts are so intricate that the normative levels are too coarse and deterministic to provide a comprehensive yet manageable picture that can be processed further by designers, occupants and decision takers. Moreover, the high specificity of such building types makes an extensive empirical analysis of existing buildings an obvious first step towards the specification of wayfinding and other simulations for use in the early design stages.

The initial application of route analysis in this framework formed part of a wider analysis of ten Dutch prisons in 1999-2000. It focused on basic routes common to all prison buildings that have substantial contribution to their management and operating costs, especially with respect to security facilities and personnel. This also makes such routes an important implicit factor in prison programming (e.g. for clustering composition and sizes), as well as for prison typology, which is based on assumptions underlying the (standardized) prison brief. Pedestrian circulation is furthermore important for user satisfaction. While the preferred length of route is a matter of prison regime and approach to supervision, complexity and lack of overview and control in the movement of prisoners is always undesirable.

The building representation of the prisons serves as basis for the topological registration of the routes as sequences of spaces linked by doors and other access-permitting openings. The topological representation is complemented by a geometric one that allows for a higher accuracy in the description of the route path. Input and output relations between route and building representations are largely automated, including production of the geometric route locally (i.e. within each space) and measurement of route distance and complexity. Use data (purpose of route, user and temporal information) are collected in alphanumeric databases and linked dynamically to the route representation. Also the analyses of routes individually and comparative analyses of different routes and different buildings in spreadsheets are linked dynamically to the spatial representations. 


\section{DESCRIBING A ROUTE TOPOLOGICALLY}

A pedestrian route between two spaces $A$ and $B$ can be described topologically by the sequence of spatial elements that must be traversed so as to travel from $A$ to $B$. This sequence comprises spaces and doors or similar openings permitting access. Consequently, a route can be represented by a subset of a building representation at two correlated levels, the topological and the geometric (Tabor, 1976). Topological route representations are closely associated with wayfinding. The basis of topological wayfinding is the access graph of a building, which is transformed into search tree by tracing all possible paths to the point they reenter, previously visited nodes. Topological search for a path comes in two variations:

- Satisfactory paths: finding paths from starting positions to destinations by means of simple, basic procedures such as depth-first or breadth-first search. The length of the paths is generally not important.

- Optimal paths: finding shortest paths with procedures such as branch and bound, discrete dynamic programming or $\mathrm{A}^{*}$. In these the cost of traversing a path is of primary importance.

An interesting extension of the optimal paths variation is wayfinding with adversaries, as in e.g. games, by means of procedures such as minimax (Shannon, 1950) and alpha-beta pruning (Knuth and Moore, 1975). These provide possibilities for relating pedestrian movement with its context (intervening opportunities).

Also in our system the topological representation of a route is the starting point of registration and analysis. Rather than calculating the path on the basis of starting point and destination, the route data were registered in visits to the buildings and in interviews with the route users and controllers. The actual routes exhibit several marked differences with normative paths. The most important is that actual routes may contain loops. This is consistent with the security and supervision constraints of a prison. Loops in routing also exist in other building types such as exhibition spaces and should not be approached as a mismatch between circulation requirements and spatial arrangement. On the contrary, they can be features of specific activities. Another difference between the actual routes and some wayfinding and clustering approaches is that a route and its path are oriented: pedestrian circulation from $A$ to $B$ is not equivalent to the circulation from $B$ to $A$. The removal of orientation that makes many formulations of a brief or of pedestrian circulation hopelessly static is not applicable to route analysis.

The implementation of the topological route representation posed a practical problem. While it derives from the tradition of graph-based representations of built space, the use of graphs on top of the building 
representation would reduce the legibility of both the spatial and the route representation. Moreover, many users of the information system would have difficulty with graph representations. For these reasons, the topological representation was implemented as an alphanumeric annotation of a set of spaces (link to external database) and expressed visually as graphic annotations of the spaces by means of hatching. The sequence by which spaces are traversed in the route was not indicated in the annotation but, similarly to other relations in the representation, could be detected on an ifneeded basis by the existence of direct access between spaces in the set of a specific route. The detection of loops is not included in this version of wayfinding, as it requires closer correlation with extrinsic, yet uncharted constraints such as visual and electronic supervision.

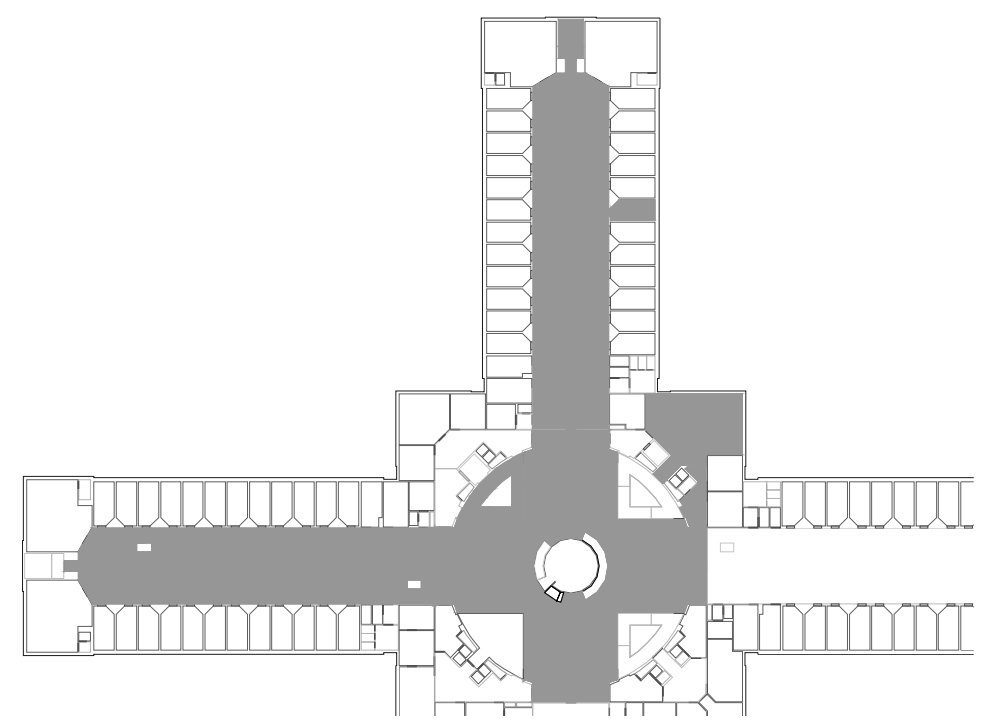

Figure 1. Topological route representation

\section{DESCRIBING A ROUTE GEOMETRICALLY}

Any elaboration of the topological representation of a route (with the exception of some temporal aspects) involves transition to a geometric representation where the path of the route is described with accuracy and precision. The geometric representation is obviously more significant within the nodes of a topological representation, i.e. within the spaces of the route. The size of most doors and similar openings is such that any perturbation of the path as it passes though them is insignificant for the overall route. This does not preclude local ergonomic or cognitive problems, e.g. in opening a 
door. However, such problems depend more on detailing and are generally as invisible in the geometric representation as in the topological one. Alphanumeric or other annotations are probably the best means for registering these problems on the route representations.

The geometric path in a space has been a traditional hard problem in both wayfinding and route analysis. The usual focus has been obstacle avoidance. Nevertheless, we should not forget the influence of intervening opportunities of e.g. interaction with the building and its occupants, as well as relations with other routes that may cause congestion or disorientation. As a result, models of spatial reasoning, i.e. reasoning about motion in space have to move beyond avoiding obstacles and moving around obstacles (Brady, Hollerback et al., 1982; Brooks, 1983; Lozano-Pérez, 1983) to be really applicable in the analysis of buildings and designs. Recent research into the applicability of autonomous mechanisms and interactive immersive environments for wayfinding seems promising in this respect (Shih and Yan, 1997; Dijkstra, Timmermans et al., 2000).

In a conscious attempt to reduce the complexity of their subject matter, conventional analyses of pedestrian circulation have employed approximations of the path by means of techniques such as the straight line or the city block. In our analyses ascertaining the actual path is also too timeconsuming and uncertain to be a viable option. Even under the carefully controlled conditions of a prison, human choice and contextual variability conspire to create a spectrum of local alternatives and variations. For such reasons we have opted for a normative basis derived from the definition of the shortest path in current Dutch building regulations, which has been previously applied to fire escape analysis (Koutamanis, 1995). The essential characteristic of this definition with respect to obstacle avoidance is that the shortest path should have a distance of at least thirty centimetres from fixed obstacles. Also the starting point of a path should be the furthest point from the exit in the departure space, again thirty centimetres away from fixed obstacles such as walls.

Our basic adaptation of the normative definition attempts to neutralize the slight bias towards the destination side that results from the thirtycentimetre constraint. The starting point is assigned to the centroid of the departure space and the path passes openings in the middle. All other obstacles were kept at a distance of thirty centimetres. The user is allowed to add to the regulatory fixed obstacles, so as to improve the precision of the average route path. The resulting geometric representation elaborates locally the connection of two links in the topological representation through the connected node: the local path starts at the centroid of the first link (opening) and ends at the centroid of the other link, passing though the node (space) 
and keeping within this space a distance of thirty centimetres from all objects that should be treated as obstacles.

The explicit representation of relevant entities in the building representation and the use of local intelligence permits a large degree of automation in the derivation of the route paths. User input is limited to the selection of the two openings that should be linked by a local path and this only if a space has more than two openings. The only other possibility for user interaction concerns obstacle avoidance: if a path comes closer than thirty centimetres to an obstacle, the route analysis system does not attempt a shortest path calculation around the obstacle but refers the problem to the user. With the initial path and its intersections with a thirty-centimetre no-go area around the obstacle as basis, the user traces over the local path that agrees most with the actual route.

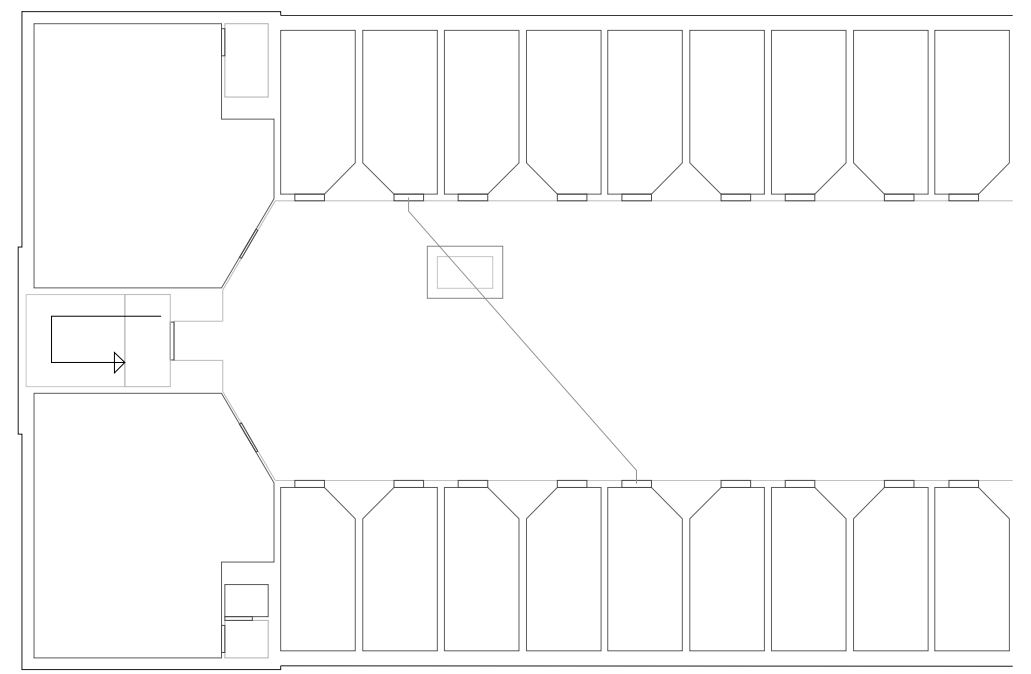

Figure 2. Local path and obstacle in the geometric route representation

Also the integration of local paths into the complete geometric representation of a route is based on local recognition. As local paths connect with each other at the centroids of openings, the topological representation is used to collate them together into the geometric representation of the route.

Non-horizontal paths are often treated as a weighted version of their horizontal projection. However, a prerequisite to the use of coefficients for the adaptation of distance, time and energy spent along a non-horizontal path of the route is justification of the ergonomic and cognitive adaptation factors and validation of the results. The lack of such data meant that we opted for a 
geometrically accurate three-dimensional representation of the path that allowed for the identification of vertical as well as horizontal displacement along the route.

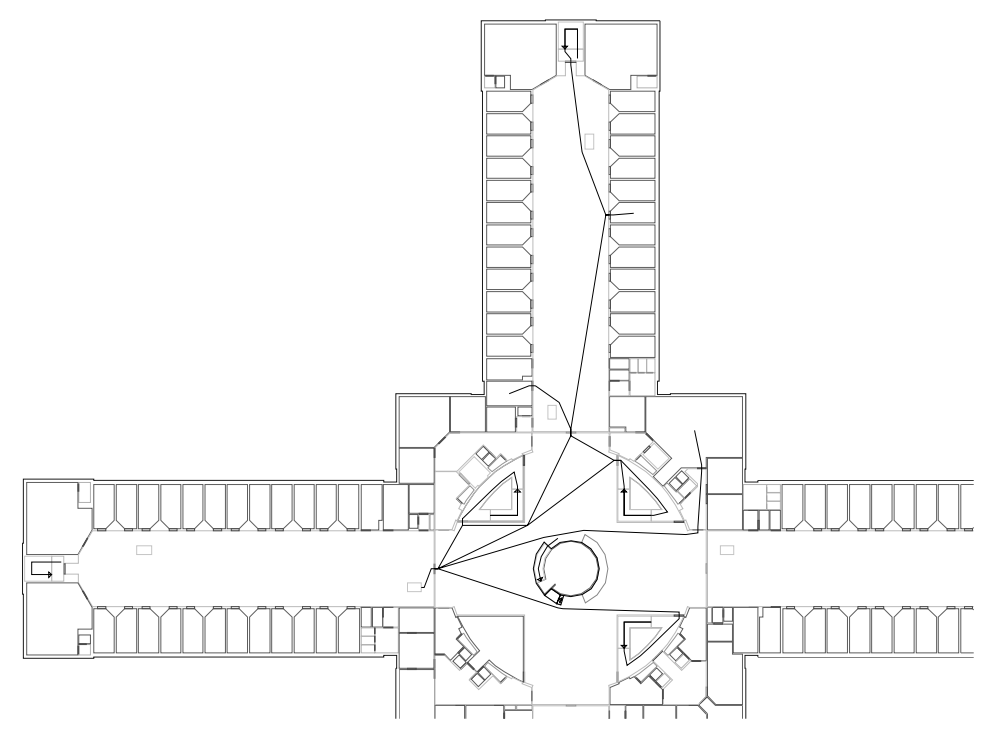

Figure 3. Geometric route descriptions

\section{FURTHER DEVELOPMENT}

The route analysis system described in the present paper is primarily meant as an information-management component of a wider design information system. In its current form it provides a reasonably accurate and comprehensive description of pedestrian circulation in quite complex buildings. Moreover, this description is at an appropriate level of abstraction for serving as communication matter in the discussions between designers, decision takers and building users. In the framework of the prison analysis of 1999-2000 it was used effectively to assess pedestrian circulation in each prison individually and to compare similar routes in different buildings. The ability to analyse and compare with respect to both the topology and the geometry of routes, allowed for different measures of complexity and distance. Moreover, the combination of qualitative and quantitative data facilitated the exploration and presentation of the effects of wider programming and typologic constraints on prison design in The Netherlands. Such effects are not a matter of a global measure of circulation cost but of precise identification and substantiation of observed and potential problems, both within a route and in the relations between separate routes. In existing 
buildings an accurate description of such problems provides a responsive analysis of ideas concerning the improvement of facilities management, activity allocation, operational cost reduction and redevelopment of a building. The same information can also influence the design of similar new buildings as a source of examples for brief improvement and testing, as well as of cases for refinino decion thinkino

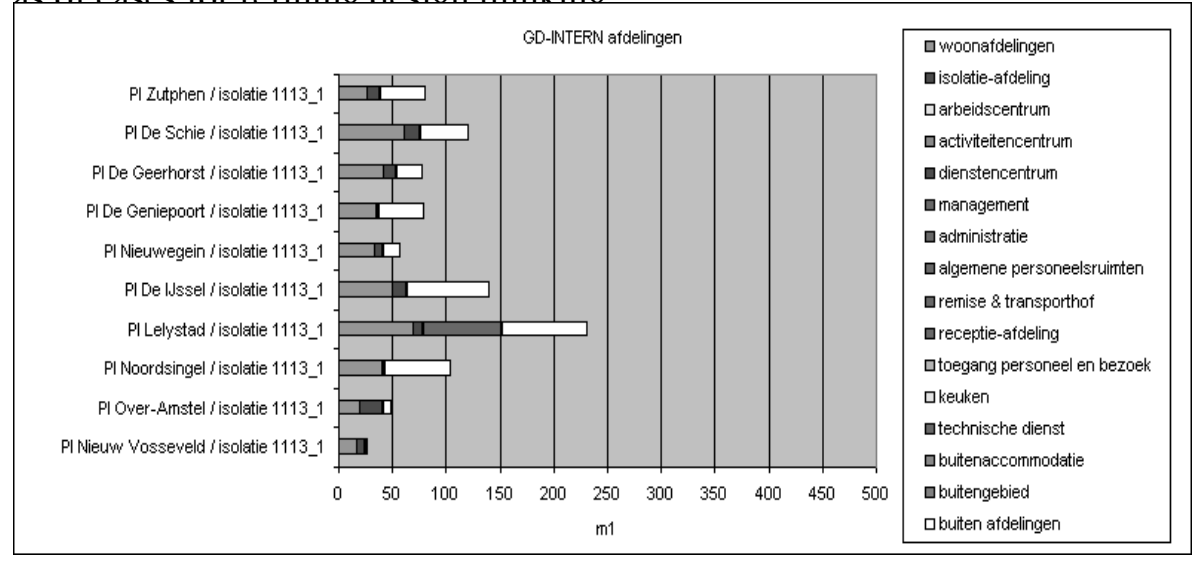

Figure 4. Route comparison

From a methodical and technical viewpoint, the route analysis system provided useful insights into the possibilities for adapting wayfinding research results to the prediction of realistic routes. For example, the effects of compartmentalization, security barriers and heuristics for e.g. avoiding spatio-temporal coincidence of distinct routes are such that automatic shortest path calculation either becomes too complex and therefore too unreliable without user intervention. A promising direction for further development is he addition of memory components that represent knowledge of the specific building (Gross and Zimring, 1992), as well as of the particular rules and constraints of prison supervision and security.

Such memory components relate closely to local intelligence and in particular to autonomous mechanisms that reproduce or explore wayfinding behaviour in the building representation. Prior experience with such mechanisms suggests that they are helpful for the representation of temporal aspects and for the exploration of capacity issues (Koutamanis, 1995) but require a more global knowledge of the building and the route in order to go beyond local refinement. For example, finding an optimal path in a route with intervening opportunities and conflicts presupposes reliable avoidance of the horizon effect, i.e. recognition of lesser, temporary sacrifices in the context of wider concerns and gains (Berliner, 1978; 1980).

On a practical level, further development of the route analysis system should address its two major limitations for information processing: 
1. The temporal dimension (including speed of movement), which is currently absent from the system not in terms of registrable information but of simulation possibilities that facilitate comprehension and communication of pedestrian circulation patterns.

2. Representation of capacity and volume in the routes and the sequence of spaces and openings that accommodate them. These are arguably the most important omissions in the current information system, as they provide essential indications of functional interaction with building and related performance measures.

\section{REFERENCES}

Berliner, H.J. (1978). “A chronology of computer chess and its literature.” Artificial Intelligence 10(2).

Berliner, H.J. (1980). “Computer backgammon.” Scientific American (June).

Blue, V.J. and J.L. Adler (1998). "Emergent fundamental pedestrian flows from cellular automata microsimulation." Transport Research Board (1644): 29-36.

Brady, M.J., J.M. Hollerback, et al., Eds. (1982). Robot motion: planning and control. Cambridge, Massachusetts, MIT Press.

Brooks, R.A. (1983). "Planning collision free motions for pick and place operations." International Jurnal of Robotics Research 2(4).

Dijkstra, J., H.J.P. Timmermans, et al. (2000). Towards a multi-agent system for visualizing simulated behaviour withing the built environment. Design and decision support systems in urban planning. Proceedings of the 5th International Conference. Eindhoven, Eindhoven University of Technology.

Gross, M.D. and C. Zimring (1992). Predicting wayfinding behavior in buildings: a schemabased approach. Evaluating and predicting design performance. Y. E. Kalay (ed.). New York, Wiley.

Knuth, D.E. and R.W. Moore (1975). "An analysis of alpha-beta pruning.” Artificial Intelligence 6(4).

Koutamanis, A. (1995). Multilevel analysis of fire escape routes in a virtual environment. The global design studio. M. Tan and R. The (eds.). Singapore, Centre for Advanced Studies in Architecture, National University of Singapore.

Koutamanis, A. (2000). "Digital architectural visualization." Automation in Construction 9(4): 347-360.

Koutamanis, A. and V. Mitossi (1993). On the representation of dynamic aspects of architectural design in machine environment. Advanced technologies. Architecture, planning, civil engineering. M. R. Beheshti and K. Zreik (eds.). Amsterdam, Elsevier.

Koutamanis, A. and V. Mitossi (1996). Simulation for analysis: Requirements from architectural design. Full-scale modeling in the age of virtual reality. B. Martens (ed.). Vienna, OKK.

Koutamanis, A. and V. Mitossi (2000). Grammatical and syntactic properties of CAAD representations for the early design stages. Design and decision support systems in architecture. Proceedings of the 5th International Conference. Eindhoven, Eindhoven University of Technology. 
Leusen, M.v. and V. Mitossi (1998). A practical experiment in representation and analysis of buildings. 4th Design and Decision Support Systems in Architecture and Urban Planning Conference. Eindhoven.

Lozano-Pérez, T. (1983). "Spatial planning: A configuration-space approach.” IEEE Transactions on Computers 71(7).

Marchant, E.W. (1980). Modelling fire safety and risk. Fires and human behaviour. D. Canter. Chichester, Wiley.

Mitossi, V. and A. Koutamanis (1998). Spatial representations as the basis of formal and functional analysis. 4th Design and Decision Support Systems in Architecture and Urban Planning Conference. Eindhoven.

Ozel, F. (1992). An intelligent simulation approach in simulating dynamic processes in architectural environments. CAAD Futures ' 91 —Computer Aided Architectural Design Futures. Education, research, applications. G.N. Schmitt. Wiesbaden, Vieweg.

Shannon, C.E. (1950). "Automatic chess player." Scientific American 182(48).

Shields, T.J. and G.W.H. Silcock (1987). Buildings and fire. Harlow, Essex, Longman.

Shih, N.-J. and C.-S. Yan (1997). A study of the location of fire egress signs by VR simulation. CAAD Futures 1997. R. Junge (ed.). Dordrecht, Kluwer.

Stollard, P. and J. Abrahams (1995). Fire from first principles. A design guide to building fire safety. London, E. \& F. Spon.

Tabor, P. (1976). Analysing route patterns. The architecture of form. L. March (ed.). Cambridge, Cambridge University Press.

Winston, P.H. (1992). Artificial Intelligence. Reading, Massachusetts, Addison-Wesley. 